\title{
Epothilones in epithelial ovarian, fallopian tube, or primary peritoneal cancer: a systematic review
}

This article was published in the following Dove Press journal:

OncoTargets and Therapy

20 August 2015

Number of times this article has been viewed

Flora Zagouri'

Theodoros N Sergentanis ${ }^{2}$

Dimosthenis Chrysikos ${ }^{2}$

Meletios-Athanassios

Dimopoulos'

Aristotle Bamias'

'Department of Clinical Therapeutics, Alexandra Hospital, ${ }^{2}$ First

Propaedeutic Surgical Department,

Hippokration Hospital, University of

Athens, Athens, Greece
Correspondence: Flora Zagouri

Department of Clinical Therapeutics,

Alexandra Hospital, University of Athens,

Vasilissis Sofias Avenue and Lourou

Street, Athens II52I, Greece

$\mathrm{Tel}+30213216254$ I

$\mathrm{Fax}+302132162511$

Email florazagouri@yahoo.co.uk
Abstract: Ovarian cancer is the most lethal gynecologic malignancy; consequently, there is a need for effective therapies. Epothilones are microtubule-stabilizing agents that inhibit cell growth. Currently, patupilone and its four synthetic derivatives ixabepilone, BMS-310705, sagopilone, 20-desmethyl-20-methylsulfanyl epothilone B and epothilone D, as well as its derivative KOS-1584, are under clinical evaluation. This is the first systematic review conducted in accordance with the PRISMA (Preferred Reporting Items for Systematic Reviews and MetaAnalyses) guidelines that synthesizes all available data emerging from trials and evaluates the efficacy and safety of epothilones in epithelial ovarian, primary fallopian tube, and primary peritoneal cancer. Despite the fact that epothilones have proven active in taxane-resistant settings in preclinical models, it is not yet clear from Phase II/III studies reviewed here that their clinical activity is superior to that of taxanes. Nevertheless, responses to epothilones have been observed in platinum-refractory/resistant ovarian cancer patients. Moreover, despite the shared mechanism of action of epothilones, their clinical profile seems clearly different, with diarrhea being the most common dose-limiting toxicity encountered with patupilone, whereas neutropenia and sensory neuropathy are the most common toxic effects observed with the other epothilones. In any case, randomized trials comparing epothilones with standard treatments seem warranted to define further the role of these agents, whereas biomarker analysis might further optimize patient selection.

Keywords: ovarian cancer, epothilone, patupilone, ixabepilone, systematic review

\section{Introduction}

Ovarian cancer is the fifth-most common cause of cancer death in women and the most lethal gynecologic malignancy. ${ }^{1,2}$ In 2012, it was estimated that 22,280 women would be diagnosed with and 15,500 women die of ovarian cancer in the US. ${ }^{3}$ The overall 5-year survival for 2002-2008 in 18 Surveillance, Epidemiology, and End Results geographic areas was $43.7 \% .^{3}$ Therefore, ovarian cancer might represent an important public health issue. Landmark studies, such as GOG-111 and GOG-114, have established a platinum-taxane combination as the standard chemotherapy treatment for epithelial ovarian cancer. ${ }^{1,2}$ However, more than $50 \%$ of patients with advanced disease (International Federation of Gynecology and Obstetrics stage III and IV) will relapse, requiring second-line treatment. Consequently, there is an urgent need for effective therapies for patients with relapsed ovarian cancer, particularly platinumresistant disease.

Epothilones are microtubule-stabilizing agents that inhibit cell growth. ${ }^{4,5}$ They bind to the $\beta$-tubulin subunit of the $\alpha \beta$-tubulin dimer of microtubules and induce microtubule polymerization and stabilization, resulting in $\mathrm{G}_{2} / \mathrm{M}$ arrest and the induction of apoptosis. ${ }^{4,5}$ Epothilones are less susceptible than taxanes to overexpression of P-glycoprotein, the presence of certain tubulin isoforms (class III $\beta$-tubulin), and 
tubulin mutations, all of which have been implicated in taxane resistance. ${ }^{6,7}$ Although epothilones share a similar mechanism of action with the taxanes, they are structurally unrelated. They are 16-member ring macrolides that are combined with a methylthiazole side chain. ${ }^{8}$ Naturally occurring epothilones are classified as either epoxides (epothilones A, B, E, and F) or olefins (epothilones C and D). ${ }^{8}$ Modifications to the chemical structure of the macrolide ring have been shown to alter its biologic activity and pharmacologic properties. ${ }^{8-10}$ Epothilones A and B have shown potent cytotoxic activity in paclitaxel-sensitive and paclitaxel-resistant cells expressing P-glycoprotein or mutant tubulin, but are inactivated via esterase cleavage. ${ }^{11}$ Moreover, they have demonstrated high activity in human ovarian tumor xenograft and syngeneic mouse models. ${ }^{12,13}$ In light of these promising nonclinical findings, it has been postulated that epothilones may be an effective anticancer treatment. Currently, patupilone and its four synthetic derivatives ixabepilone, BMS-310705, sagopilone, 20-desmethyl-20-methylsulfanyl epothilone B, and epothilone D, as well as its derivative KOS-1584, are under clinical evaluation.

This is the first systematic review of the literature aiming to synthesize all available data emerging from trials and to evaluate the efficacy and safety of epothilones in epithelial ovarian cancer. Moreover, this systematic review deals with the administration of epothilones in primary fallopian tube and primary peritoneal cancer, given that they are managed in a similar way to epithelial ovarian cancer.

\section{Search strategy and data abstraction}

This systematic review was performed in accordance with the PRISMA (Preferred Reporting Items for Systematic Reviews and Meta-Analyses) guidelines. ${ }^{14}$ The protocol of this systematic review has been submitted to the Institutional Review Board of Hippokration Hospital, Medical University of Athens, Greece, and is available upon request. Eligible articles were identified by a search of the Medline bibliographical database for the period up to September 30, 2012. The search strategy included the following keywords: ((ovarian OR ovary OR fallopian OR peritoneal OR peritoneum) AND (neoplasms OR neoplasm OR cancer OR cancers OR carcinoma OR carcinomas)) AND (epothilone OR EPO906 OR patupilone OR “BMS-247550” OR “aza-epothilone B” OR ixabepilone OR “BMS-310705” OR “KOS-862” OR “desoxyepothilone B” OR "KOS-1584" OR “ZK-EPO” OR “ZK 219477” OR sagopilone OR SH-Y03757A OR “BMS-247550").
Language restrictions were applied (only articles in English, French, and German were considered eligible); two investigators (FZ and DC), working independently, searched the literature and extracted data from each eligible study. Reviews were not eligible, while all prospective and retrospective studies, as well as case reports, were eligible for this systematic review. Manuscripts that did not state the names of the authors were excluded. In addition, we checked all the references of relevant reviews and eligible articles that our search retrieved, so as to identify potentially eligible conference abstracts.

All studies that examined the efficacy and safety of epothilones in epithelial ovarian, primary fallopian tube, or primary peritoneal cancer and reported the relevant frequencies, regardless of sample size, were considered eligible for this systematic review. For these studies, the following data were collected: first author, year of publication, agents, phase of the trial, number of patients treated, characteristics of patient population (first-line, second-line treatment, platinum-resistant, platinum-sensitive, etc), median age (years), complete response (CR) rate, partial response (PR) rate, stabilization of the disease (SD) rate, progression of the disease (PD) rate, median overall survival (OS) in months, median progression-free survival (PFS) in months, and complications. In instances where multiple (overlapping) publications stemming from the same study were identified, the larger-size study was included, unless the reported outcomes were mutually exclusive.

\section{Results}

The search strategy retrieved 74 articles. Of these articles, 44 were irrelevant, 17 were reviews, and 13 were eligible. ${ }^{15-27}$ After a search of the references of all reviews and remaining articles, 14 additional conference abstracts/articles were also included. ${ }^{28-41}$ Overall, 27 studies (1,293 patients) were eligible for the systematic review (Table 1). ${ }^{15-39}$ The aforementioned steps of the selection process are illustrated in detail in Figure 1.

There were seven studies evaluating patupilone $(1,046$ patients). ${ }^{15,17,21,22,24,34,35}$ Five studies described the results of Phase I trials, ${ }^{17,21,22,24,35}$ one those of a Phase II trial, ${ }^{34}$ and one study involved a Phase III design. ${ }^{15}$ Patupilone has been evaluated as monotherapy ${ }^{15,17,21,24,34,35}$ or combined with carboplatin in epithelial ovarian, primary fallopian, or primary peritoneal cancer. ${ }^{22}$ Three studies were conducted on platinum-refractory or -resistant patients, ${ }^{15,21,34}$ while in one study separate data for platinum-sensitive and platinum-refractory or -resistant patients were provided; ${ }^{22}$ in three studies, no data were provided regarding platinum sensitivity. ${ }^{17,24,35}$ The overall response rate 


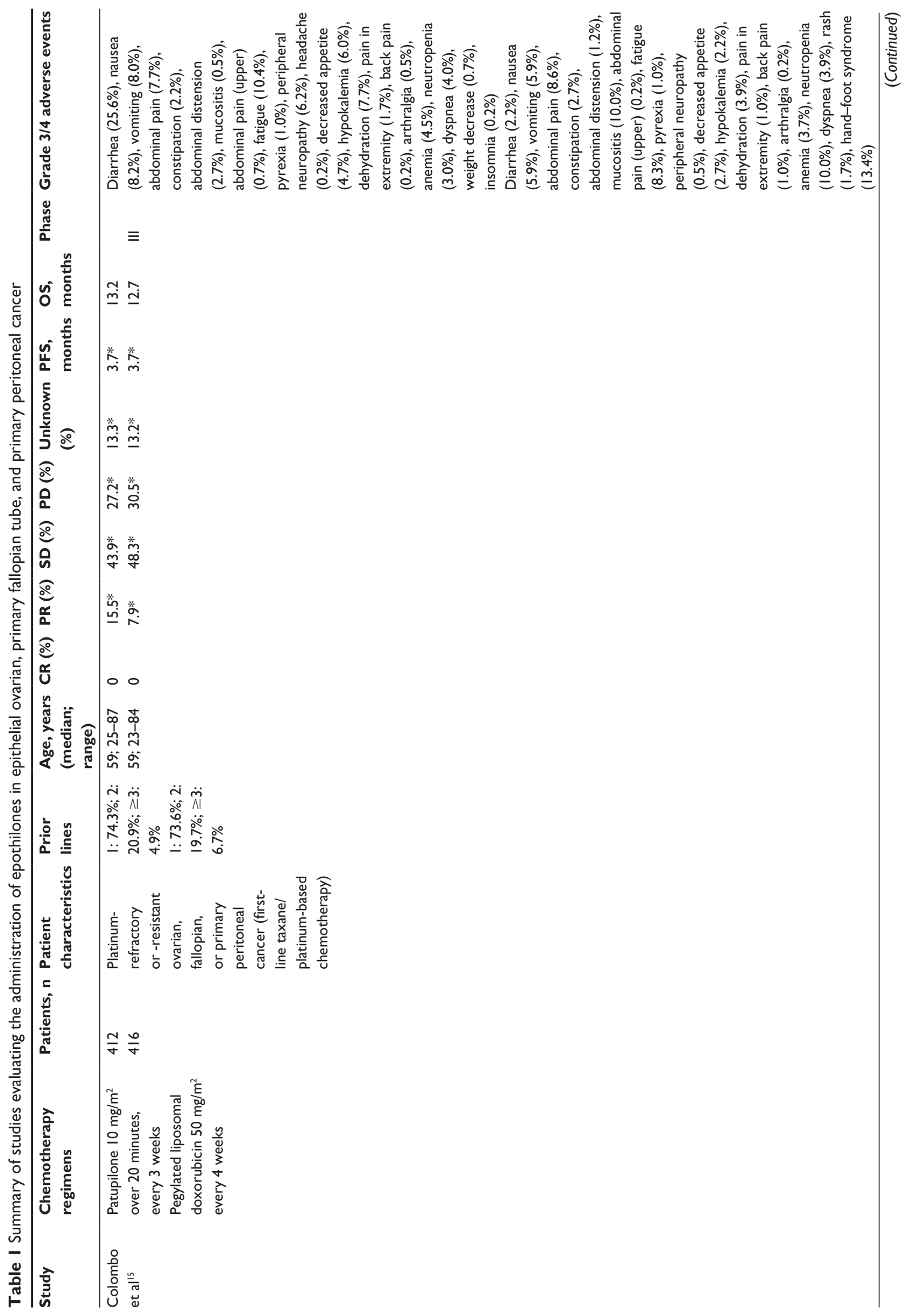




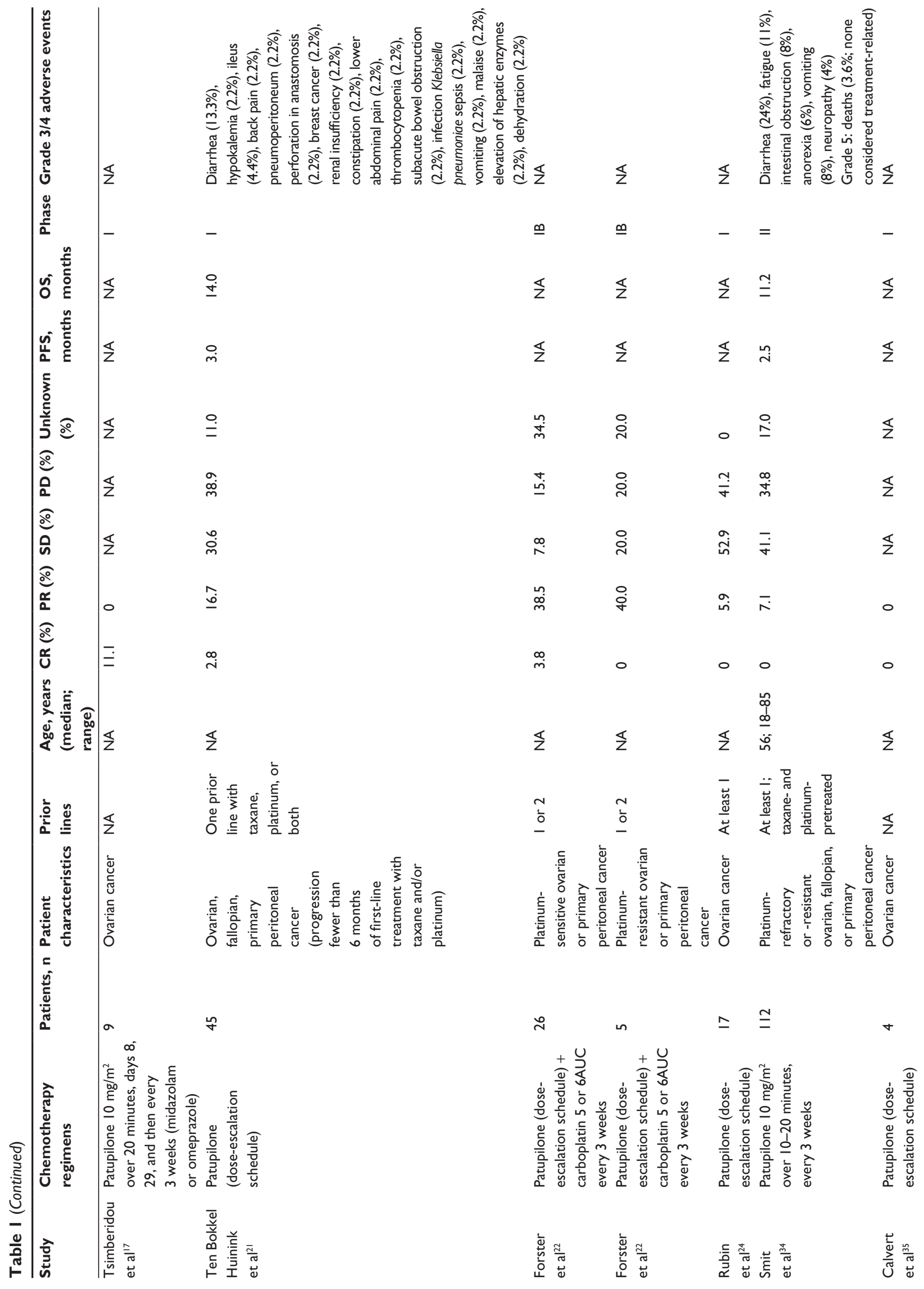




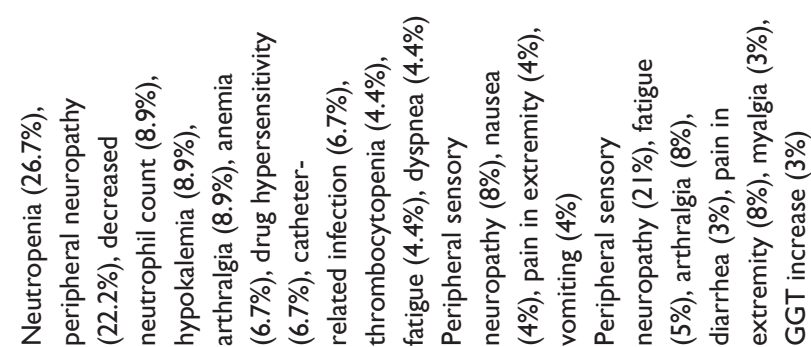

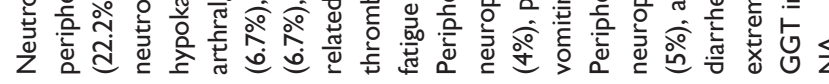

इ

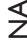

$\stackrel{m}{n} \stackrel{m}{r}$

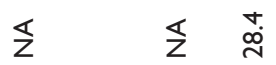

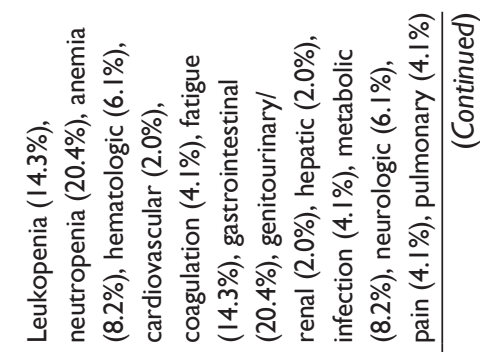

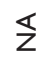

సิ กิ

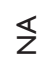

$\S \leftleftarrows$

$\underset{\infty}{\infty}$

iั

$\circ \quad 0 \quad \mathbb{Z}$

$\stackrel{\infty}{i}$

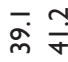

ㅇ.

○ $\longleftarrow$

సิ

迎

고 ำกำ

$\stackrel{\circ}{\circ}$

으 §

$\stackrel{\infty}{\circ}$

웅

$\overline{\underline{m}} \dot{+}$

- $\quad \stackrel{\stackrel{n}{n}}{\sim}$

$\stackrel{\sim}{\infty}$

$\underset{\infty}{m}$

$\circ \circ$

- 0 คे

$\overline{6}$

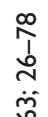

i

岁㖗

艺芒

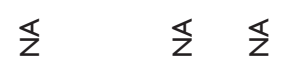

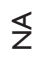



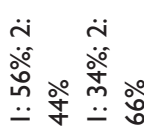

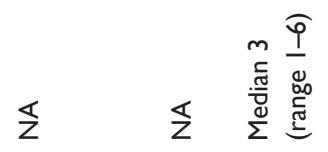

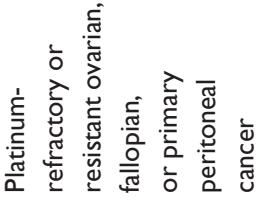

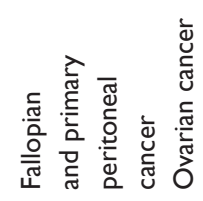

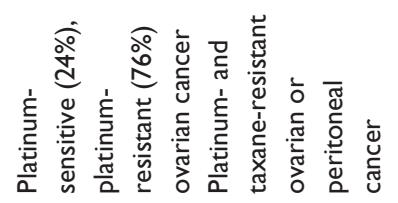

๒

$\stackrel{\infty}{m}$

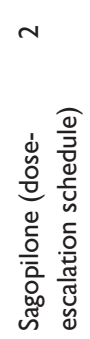

$-1$

q

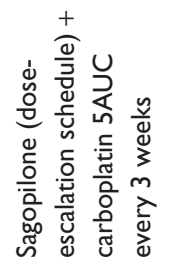

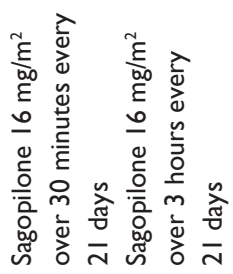

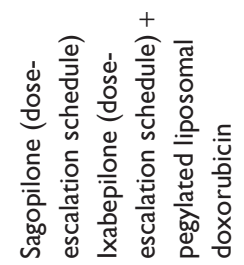

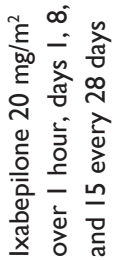

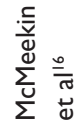

紊

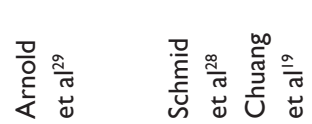

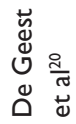




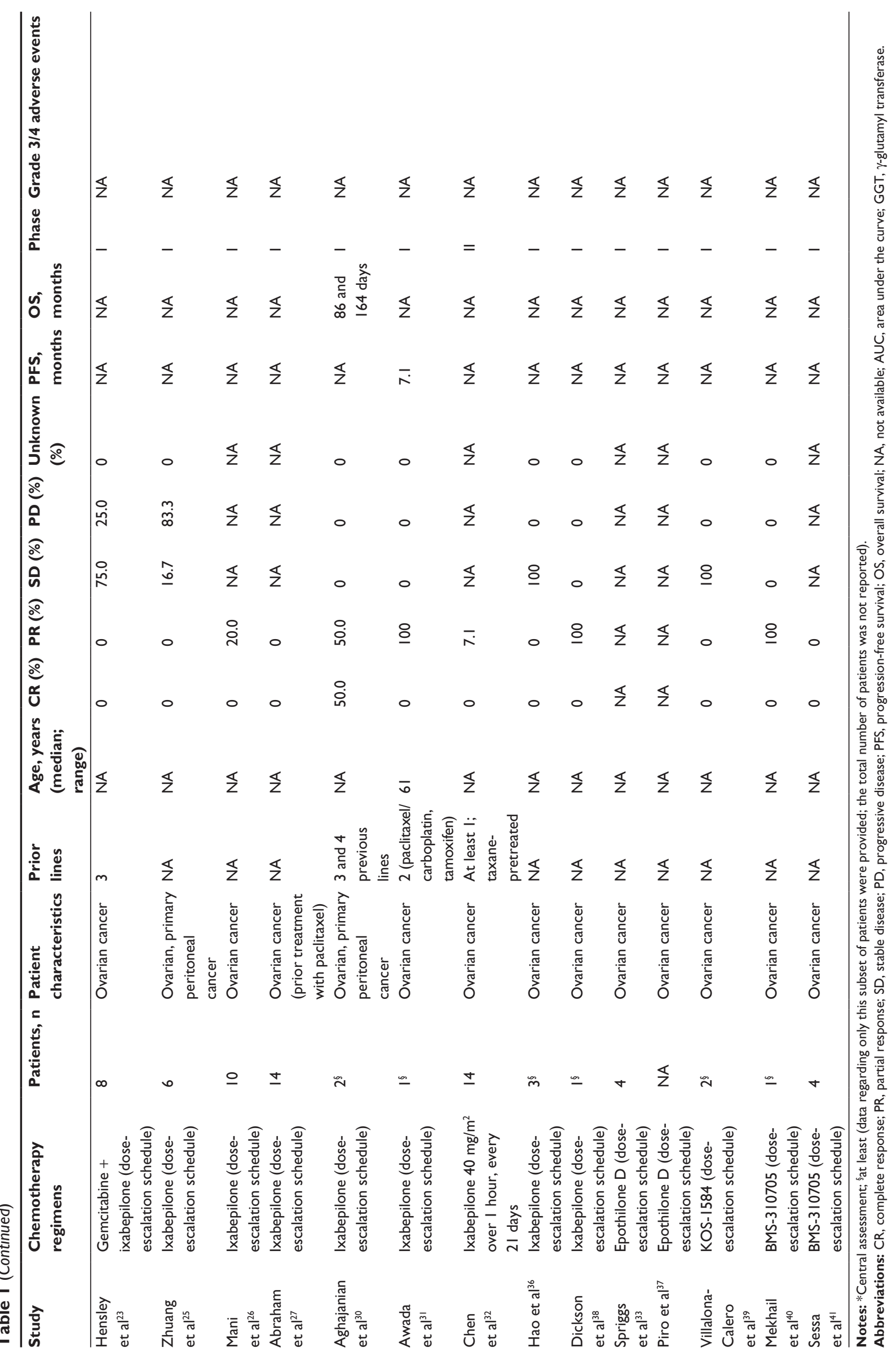




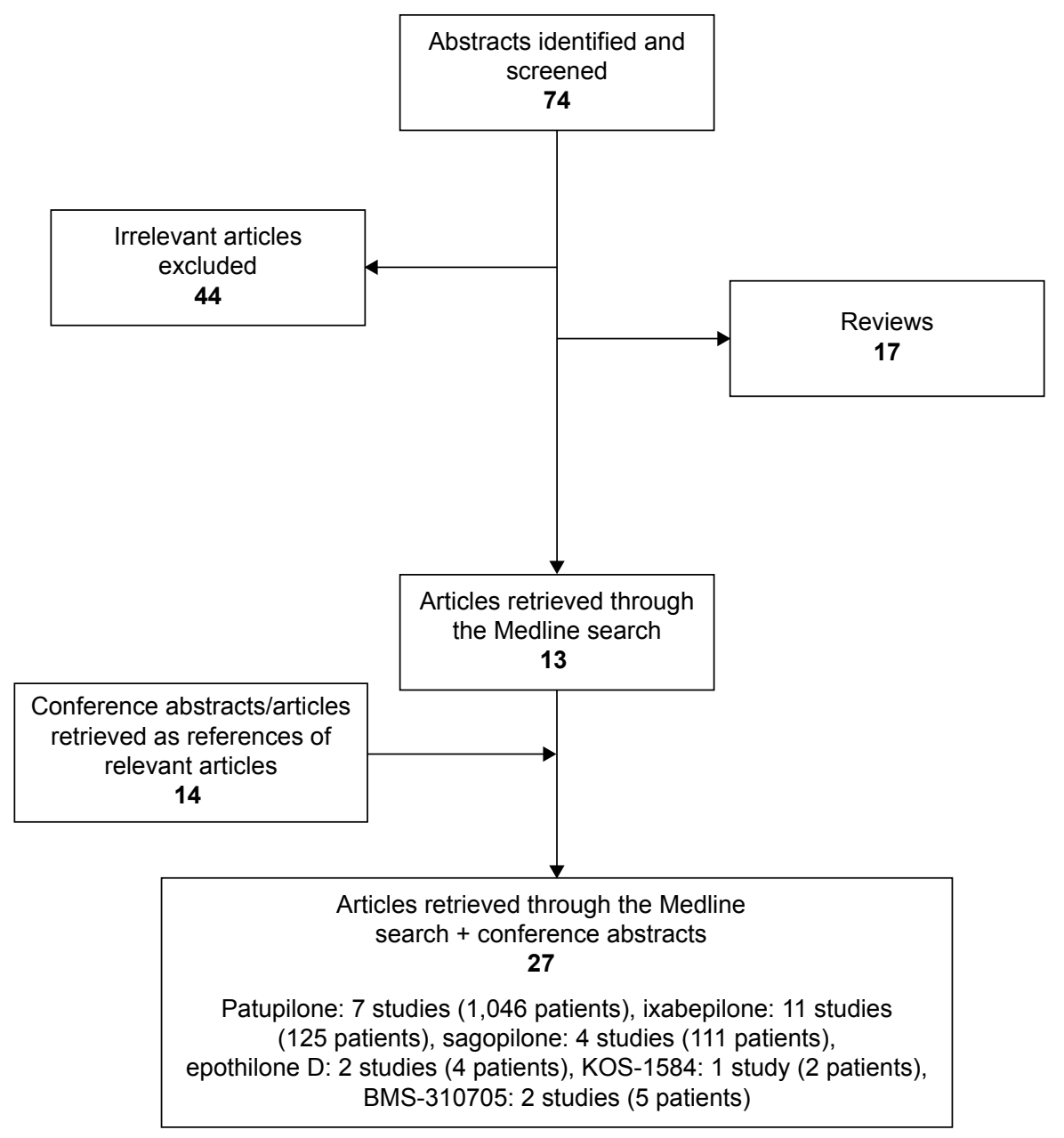

Figure I Stages of the search strategy.

(ORR) ranged between 0 and $42.3 \%$ in all studies. . $^{15,17,21,22,24,34,35}$ Data regarding the median PFS and OS were reported in three studies: ${ }^{15,21,34}$ the median PFS ranged between 2.5 and 3.7 months, while the median OS ranged between 11.2 and 14.0 months. ${ }^{15,21,34}$ All studies reported detailed outcomes pertaining to efficacy and/or safety. ${ }^{15,17,21,22,24,34,35}$ Further details are provided in Tables 1 and 2.

With regard to ixabepilone, eleven studies (125 patients) were identified, ${ }^{19,20,23,25-27,30-32,36,38}$ nine described the results of Phase I trials, ${ }^{19,23,25-27,30,31,36,38}$ and two the results of nonrandomized Phase II trials..$^{20,32}$ One trial dealt with platinum-refractory or -resistant epithelial ovarian cancer and primary fallopian or primary peritoneal cancer, ${ }^{20}$ while in the remaining studies no detailed data were provided. ${ }^{19,23,25-27,30-32,36,38}$ Moreover, ixabepilone has been evaluated as monotherapy, ${ }^{20,25-27,30-32,36,38}$ as well as in combination with gemcitabine ${ }^{23}$ and pegylated liposomal doxorubicin (PLD). ${ }^{19}$ Clinical data regarding ixabepilone have presented promising results, with a clinical benefit rate ranging between 0 and $100 \%$. $^{19,20,23,25-27,30-32,36,38}$
Data regarding PFS and/or OS were reported in four trials (OS 28.4-14.8 months; disease-free survival 7.1-4.1 months). ${ }^{19,20,30,31}$ All studies reported outcomes pertaining to efficacy and/or safety. ${ }^{19,20,23,25-27,30-32,36,38}$ Further details are provided in Tables 1 and 2 .

There were four studies examining sagopilone (111 patients); ${ }^{16,18,28,29}$ in three of them, the agent was evaluated as monotherapy, ${ }^{18,28,29}$ whereas in one sagopilone was tested in combination with carboplatin. ${ }^{16}$ Two studies described the results of a Phase I trial, ${ }^{28,29}$ one those of a Phase I/II trial, ${ }^{16}$ and one study involved a Phase II design. ${ }^{18}$ One trial was conducted in platinum-sensitive epithelial ovarian, primary fallopian, or primary peritoneal cancer, ${ }^{16}$ one in platinum-refractory or -resistant cancer, ${ }^{18}$ and no relevant data were provided in the other two studies. ${ }^{28,29}$ ORR ranged between 0 and $58.3 \%$, while the percentage of SD ranged between $21.7 \%$ and $100 \%$ in all studies. ${ }^{16,18,28,29}$ Data regarding the median PFS and the median OS were provided in only one study. ${ }^{18}$ All studies reported detailed outcomes pertaining to efficacy and/or safety (Tables 1 and 2). ${ }^{16,18,28,29}$ 


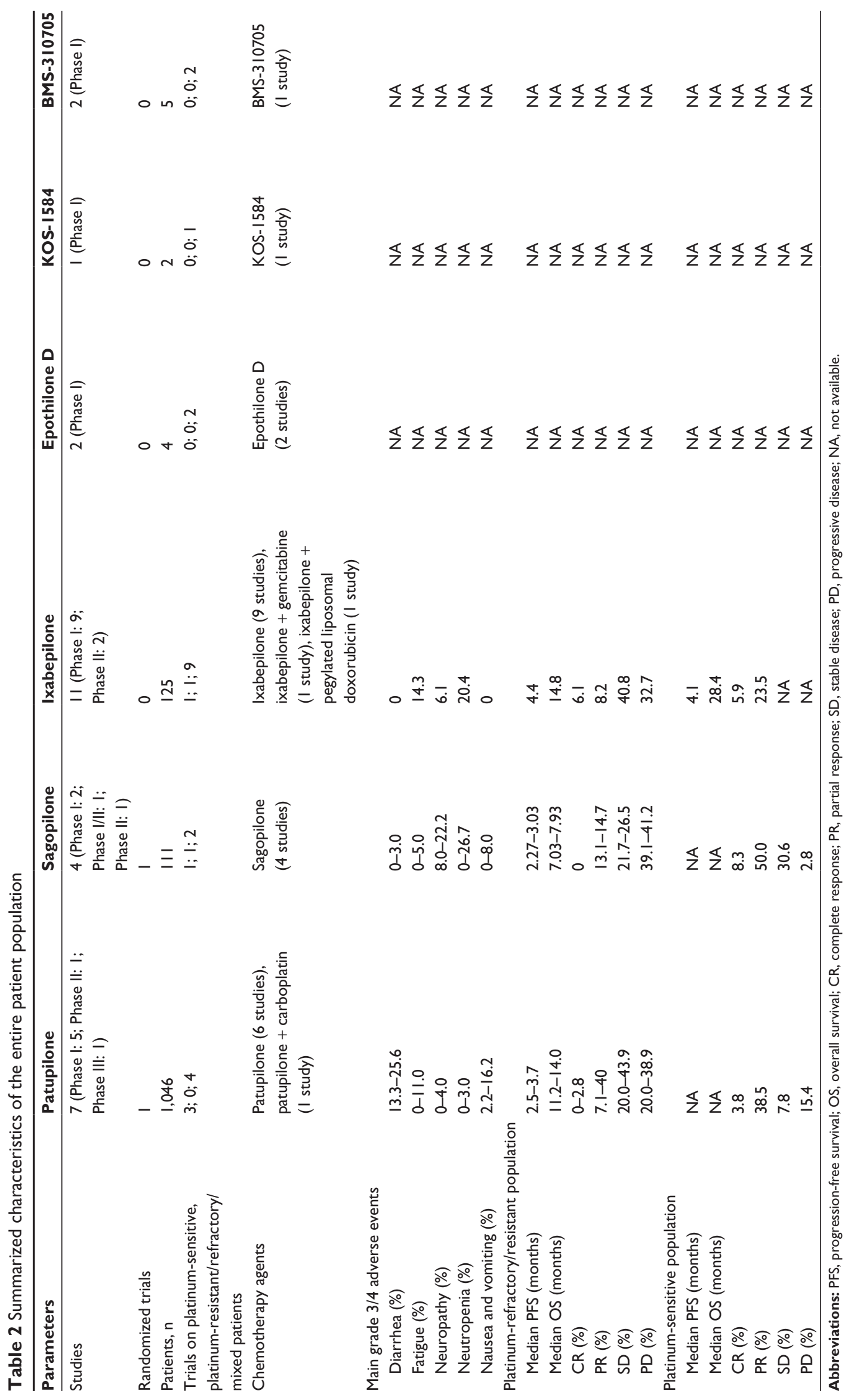


With regard to epothilone D, two studies with Phase I design were retrieved. ${ }^{33,37}$ No data regarding CR, PR, SD, PD, PFS, OS, or detailed adverse events were reported (Tables 1 and 2). Moreover, two Phase I trials (five patients) were found, evaluating BMS-310705 in ovarian cancer patients. ${ }^{40,41}$ One of these patients experienced PR, whereas detailed data regarding RRs, OS, PFS, and adverse events were not provided. ${ }^{40,41}$ As far as KOS-1584 is concerned, one Phase I study (two patients) was retrieved, ${ }^{39}$ showing SD in both patients with ovarian cancer. No data regarding PFS, OS, or adverse events were presented.

The characteristics of the individual studies are provided in Table 1, while the summarized features of the entire patient population are shown in Table 2. Qualitative interpretation and a critical, detailed evaluation of the individual eligible studies follow.

\section{Discussion}

Preclinical in vitro data, stemming from a variety of taxanesensitive and -resistant cell culture models ${ }^{5,13}$ together with in vivo findings in murine xenograft tumor models, have suggested that epothilones might be useful for the treatment of epithelial ovarian, primary fallopian, or primary peritoneal cancer. ${ }^{42,43}$ Therefore, epothilones were evaluated in the clinical setting.

\section{Patupilone (epothilone B; EPO960)}

Patupilone (epothilone B; EPO960) is a natural product. Based on Phase I/II data, a dose of $10 \mathrm{mg} / \mathrm{m}^{2}$ every 3 weeks seems to be the optimal schedule for patupilone. Doselimiting toxicities include diarrhea and fatigue, whereas neurotoxicity has also been noted. Diarrhea grade $3 / 4$ was the most common serious adverse event, ranging from $13.3 \%$ to $25.6 \%$; however, it was manageable and usually resolved without sequelae.

Regarding the platinum-refractory/resistant population, patupilone has shown promising results, with a median PFS ranging between 2.5 and 3.7 months in heavily pretreated patients, whereas the median OS ranged between 11.2 and 14.0 months (Table 2). Moreover, the ORR ranged between $7.1 \%$ and $40.0 \%$, whereas stabilization of the disease was achieved in $20.0 \%-41.1 \%$ of patients (Table 1 ).

A Phase I dose-escalation trial evaluated the safety, maximum tolerated dose, and pharmacokinetics of patupilone administered once every 3 weeks. In this trial, patupilone was well tolerated at doses up to $11.0 \mathrm{mg} / \mathrm{m}^{2}$, demonstrating promising antitumor activity (overall clinical benefit $50.1 \%$, PFS 3 months, OS 14 months). ${ }^{21}$ Adverse events were mild to moderate in intensity, with diarrhea grade $3(13 \%)$ being the most commonly reported serious adverse event. ${ }^{21}$ The maximum tolerated dose was not reached in the study, while diarrhea, peripheral neuropathy, and fatigue were the most common dose-limiting toxicities. ${ }^{21}$ The results of this trial were in accordance with those reported in a single-arm Phase II study conducted in the same patient population (platinum-refractory/resistant). ${ }^{34}$ The best ORR was $7.1 \%$, whereas $41 \%$ of patients had SD. The median PFS was 2.5 months, while the median OS was 11.2 months, with $33 \%$ of patients censored. The most common grade $3 / 4$ toxicities were diarrhea (24\%), fatigue (11\%), intestinal obstruction (8\%), and vomiting (8\%). Finally, a study by Forster et al conducted in platinum-resistant ovarian cancer patients as a Phase IB trial (ORR 40\%, SD 20\%), ${ }^{22}$ was in accordance with the aforementioned results. Of note, in this trial, patupilone was administered in combination with carboplatin.

Given the strong and promising clinical data, a Phase III trial was conducted evaluating patupilone in patients with resistant or refractory ovarian, fallopian, or peritoneal cancer. ${ }^{15}$ This study compared the efficacy and safety of patupilone $\left(10 \mathrm{mg} / \mathrm{m}^{2}\right.$ intravenously every 3 weeks) with those of PLD $\left(50 \mathrm{mg} / \mathrm{m}^{2}\right.$ intravenously every 4 weeks). There was no statistically significant difference in OS, the primary end point, between the patupilone and PLD arms (hazard ratio $0.93,95 \%$ confidence interval [CI] 0.79-1.09; $P=0.195$ ), with median OS rates of 13.2 and 12.7 months, respectively. ${ }^{15}$ Median PFS was 3.7 months for both arms. The ORR (all PRs) was higher in the patupilone arm than in the PLD arm $(15.5 \%$ vs $7.9 \%$, odds ratio $2.11,95 \% \mathrm{CI}$ 1.36-3.29), although disease-control rates were similar $\left(59.5 \%\right.$ vs $56.3 \%$, respectively). ${ }^{15}$ Frequently observed adverse events of any grade included diarrhea $(85.3 \%)$ and peripheral neuropathy $(39.3 \%)$ in the patupilone arm and mucositis/stomatitis (43\%) and hand-foot syndrome (41.8\%) in the PLD arm. ${ }^{15}$ Therefore, it seems that patupilone did not demonstrate significant improvement in OS compared with the active control, PLD.

These results led to the decision of no further development of this agent from the manufacturer. Nevertheless, a different reading of these results indicates that patupilone is active in platinum-resistant ovarian cancer, and this seems important in a chronic disease with only few active drugs being available for its platinum-resistant phase. In this context, the results of another Phase III trial evaluating patupilone vs doxorubicin in platinum-refractory/resistant ovarian, primary fallopian, or peritoneal cancer are awaited with interest (NCT00262990). 
As far as platinum-sensitive epithelial ovarian or primary peritoneal cancer is concerned, the only published data come from a Phase IB trial. ${ }^{22}$ In this trial, patupilone was combined with carboplatin in 26 patients, showing promising results (ORR 42.3\%, SD 7.8\%, PD 15.4\%). ${ }^{22}$ It seems therefore that the role of patupilone in the treatment of platinum-sensitive epithelial ovarian cancer, if any, remains to be clarified in the future. However, studies seem warranted to evaluate its potential role in this subtype of ovarian cancer, where biomarker analysis seems mandatory to further optimize patient selection.

\section{Sagopilone (ZK-EPO)}

Sagopilone (ZK-EPO) is a third-generation epothilone B. A dose of $16 \mathrm{mg} / \mathrm{m}^{2}$ over 3 hours every 3 weeks seems to be the optimal schedule. With regard to toxicity, the most common adverse events are peripheral sensory neuropathy and nausea, with neuropathy grade 3 events ranging from $8.0 \%$ to $22.2 \%$ (Tables 1 and 2). Given the promising preclinical data, as well as data emerging from Phase I trials in a wide variety of cancers (also including ovarian cancer in their pools), ${ }^{28,29}$ sagopilone has been evaluated in two trials on epithelial ovarian, primary fallopian, and primary peritoneal cancers. $^{16,18}$

More specifically, in a Phase I/II study in women with platinum-sensitive ovarian cancer, sagopilone was tested in combination with carboplatin. ${ }^{16}$ In this trial, 45 patients received sagopilone at $12 \mathrm{mg} / \mathrm{m}^{2}$ or $16 \mathrm{mg} / \mathrm{m}^{2}$. The ORR was $58.3 \%$, while the SD rate was $30.6 \%$. The main adverse events were peripheral neuropathy $(75.6 \%)$, fatigue $(71.1 \%)$, and nausea $(64.4 \%) .{ }^{16}$ Therefore, it seems that sagopilone in combination with carboplatin may be effective, whereas toxicities were manageable in patients with recurrent platinum-sensitive epithelial ovarian cancer. ${ }^{16}$ Moreover, the results from another randomized Phase II trial were encouraging. ${ }^{18}$ In this trial, patients with platinum-refractory/resistant ovarian, primary fallopian, or peritoneal cancer were randomized to receive sagopilone $16 \mathrm{mg} / \mathrm{m}^{2}$ as a 3- or 0.5-hourly intravenous infusion every 21 days for up to 6 weeks. The ORRs were $14.7 \%$ and $13.1 \%$, while the median PFS was 3.03 and 2.27 months, respectively, in both arms. The 0.5 -hour arm was closed when it failed to meet its target efficacy. The main drug-related adverse events were peripheral sensory neuropathy $(73 \%$; $16 \%$ grade 3$)$, nausea $(37 \% ; 2 \%$ grade 3$)$, fatigue $(35 \% ; 3 \%$ grade 3$)$, and arthralgia (30\%; $\%$ grade 3$)$. Overall incidence of peripheral sensory neuropathy was similar in both treatment arms, with no grade 4 neuropathy events. Therefore, it seems that sagopilone may be effective, with balanced tolerability, in patients with recurrent platinum-resistant ovarian cancer. Larger studies are nevertheless warranted to further evaluate this agent, as well as its combination with paclitaxel, in ovarian cancer patients.

\section{Ixabepilone (azaepothilone B; BMS- 247550)}

Ixabepilone (azaepothilone B; BMS-247550) is a semisynthetic analog of epothilone B. Ixabepilone has been approved by the US Food and Drug Administration in combination with capecitabine for the treatment of patients with metastatic or locally advanced breast cancer resistant to treatment with an anthracycline and a taxane, or whose cancer is taxaneresistant and for whom further anthracycline therapy is contraindicated. Moreover, it is indicated as monotherapy for the treatment of metastatic or locally advanced breast cancer in patients whose tumors are resistant or refractory to anthracyclines, taxanes, and capecitabine. The optimal schedule for ixabepilone seems to be a dose of $40 \mathrm{mg} / \mathrm{m}^{2}$ every 3 weeks. Neutropenia is the dose-limiting toxicity, with grade $3 / 4$ adverse events reaching $20.4 \%$ (Table 2 ).

Ixabepilone has been evaluated in multiple Phase I trials, where patients with ovarian cancer were included, exhibiting promising antitumor activity and a well-tolerated safety profile. ${ }^{19,23,25-27,30,31,36,38}$ Of note, in a Phase I trial, ixabepilone combined with PLD was administered in 17 patients with ovarian cancer (platinum-sensitive 24\%, platinum-resistant $76 \%$ ). ${ }^{19}$ Objective response occurred in $29 \%$ of patients (95\% CI 10\%-56\%), median PFS was 4.1 months (95\% CI 2.7-8.2 months), and median OS was 28.4 months (95\% CI 7.6 months, upper limit not reached). ${ }^{19}$

Moreover, the Phase II trial, conducted by the Gynecologic Oncology Group, evaluated the efficacy and safety of ixabepilone $\left(20 \mathrm{mg} / \mathrm{m}^{2}\right.$ administered over 1 hour on days 1 , 8 , and 15 of a 28-day cycle) in patients with recurrent or persistent platinum- and taxane-resistant primary ovarian or peritoneal carcinoma. ${ }^{20}$ The ORR was $14.3 \%(95 \%$ CI $5.9 \%-27.2 \%) .{ }^{20}$ Moreover, $40.8 \%$ of patients had SD, whereas $32.7 \%$ of them had increasing disease. The median PFS was 4.4 months (95\% CI 0.8-32.6+ months), whereas the median OS was 14.8 months (95\% CI 0.8-50.0). ${ }^{20}$ Adverse effects included peripheral neuropathy (grade 2, 28.5\%; grade $3,6.1 \%$ ), neutropenia (grade $3 / 4,20.4 \%$ ), fatigue (grade 3, 14.3\%), and nausea (grade 3, 22\%). ${ }^{20}$ The results of another Phase II trial, conducted by Chen et $\mathrm{al}^{32}$ were in agreement with the aforementioned.

It thus seems that ixabepilone may demonstrate antitumor activity and acceptable safety in patients with platinum- and 
taxane-resistant recurrent or persistent ovarian cancer. It is not clear whether this offers an advantage over retreatment with paclitaxel or docetaxel using a weekly schedule in this setting. However, larger studies of ixabepilone as monotherapy or in combination therapy in ovarian carcinoma seem warranted, with a focus on the identification of molecular markers of resistance to microtubule-stabilizing agents, including taxanes.

\section{Epothilone D (desoxyepothilone B; KOS-862)}

Epothilone D (desoxyepothilone B; KOS-862) is a synthetic epothilone, tested in various malignancies. ${ }^{33}$ Neurologic toxicity was dose-limiting in all Phase I studies of KOS-862, whereas neuropathy, fatigue, nausea, and vomiting were also observed, though to a lesser degree. ${ }^{8,33}$ Epothilone D has been evaluated in two Phase I trials. ${ }^{33,37}$ However, no data regarding adverse events, OS, PFS, or ORR were provided. Further trials are warranted to evaluate this agent in ovarian cancer patients. ${ }^{33,37}$

\section{KOS-I584 (didehydroepothilone D)}

KOS-1584 (didehydroepothilone D) is a second-generation epothilone D. The most common adverse events related with the agent are gastrointestinal disorders, fatigue, and increased aminotransferase levels. KOS-1584 has shown promising antitumor activity in two patients with ovarian cancer included in a Phase I trial, as both experienced long disease stabilization. ${ }^{39}$ However, the data regarding this agent in ovarian cancer are too limited to draw any firm conclusion; additional studies are needed.

\section{BMS-3 10705}

BMS-310705 is an aqueous soluble, semisynthetic analog of epothilone B. The most common side effects associated with this agent are neutropenia, diarrhea, and sensory neuropathy. ${ }^{40,41}$ The data on BMS-310705 in ovarian cancer patients are limited: only two Phase I trials with five patients have been published. However, one PR has been observed, showing that this agent may be active in epithelial ovarian, primary fallopian, and primary peritoneal patients. Further studies are needed to clarify the role of BMS-310705 in these patients, if any.

\section{Conclusion}

Despite the fact that epothilones have shown activity in taxane-resistant settings in preclinical models, it is not yet clear from the Phase II/III studies reviewed here that their clinical activity is superior to that of the taxanes. Nevertheless, responses to epothilones have been observed in platinumrefractory/resistant ovarian cancer patients. Moreover, it is important to note that the availability of multiple active drugs is crucial in prolonging survival in this subpopulation of ovarian cancer, which is difficult to treat. However, it should be highlighted that patupilone is not recommended for the treatment of advanced ovarian cancer in the current clinical setting outside of an investigational trial.

Moreover, despite the shared mechanism of action of epothilones, their clinical profile is clearly different, with diarrhea being the most common dose-limiting toxicity encountered with patupilone, whereas neutropenia and sensory neuropathy are the most common toxic effects observed with the other epothilones. Additionally, combination regimens with other drugs appear feasible.

In any event, randomized trials comparing epothilones with standard treatments seem more than warranted to further define the role of these agents and/or their combinations with other existing agents, whereas biomarker analysis seems mandatory to further optimize patient selection.

\section{Acknowledgment}

FZ is the recipient of a research grant from the Hellenic Society for Medical Oncology (HeSMO).

\section{Disclosure}

The authors report no conflicts of interest in this work.

\section{References}

1. Colombo N, Peiretti M, Parma G, et al. Newly diagnosed and relapsed epithelial ovarian carcinoma: ESMO clinical practice guidelines for diagnosis, treatment and follow-up. Ann Oncol. 2010;21 Suppl 5: $\mathrm{v} 23-\mathrm{v} 30$.

2. National Comprehensive Cancer Network. Epithelial Ovarian Cancer (Including Fallopian Tube Cancer and Primary Peritoneal Cancer). Version 3.2012. Fort Washington (PA): NCCN; 2012.

3. National Cancer Institute. Previous version: SEER Cancer Statistics Review, 1975-2009 (Vintage 2009 Populations). 2012. Available from: http://seer.cancer.gov/csr/1975_2009_pops09. Accessed July 23, 2015.

4. Larkin JM, Kaye SB. Epothilones in the treatment of cancer. Expert Opin Investig Drugs. 2006;15(6):691-702.

5. He L, Orr GA, Horwitz SB. Novel molecules that interact with microtubules and have functional activity similar to Taxol. Drug Discov Today. 2001;6(22):1153-1164.

6. Kowalski RJ, Giannakakou P, Hamel E. Activities of the microtubulestabilizing agents epothilones A and B with purified tubulin and in cells resistant to paclitaxel (Taxol). J Biol Chem. 1997;272(4):2534-2541.

7. Mozzetti S, Ferlini C, Concolino P, et al. Class III $\beta$-tubulin overexpression is a prominent mechanism of paclitaxel resistance in ovarian cancer patients. Clin Cancer Res. 2005;11(1):298-305.

8. Fumoleau P, Coudert B, Isambert N, Ferrant E. Novel tubulin-targeting agents: anticancer activity and pharmacologic profile of epothilones and related analogues. Ann Oncol. 2007;18 Suppl 5:v9-v15. 
9. Altmann KH, Wartmann M, O’Reilly T. Epothilones and related structures - a new class of microtubule inhibitors with potent in vivo antitumor activity. Biochim Biophys Acta. 2000;1470(3):M79-M91.

10. Watkins EB, Chittiboyina AG, Jung JC, Avery MA. The epothilones and related analogues - a review of their syntheses and anti-cancer activities. Curr Pharm Des. 2005;11(13):1615-1653.

11. Nicolaou KC, Winssinger N, Pastor J, et al. Synthesis of epothilones A and B in solid and solution phase. Nature. 1997;387(6630):268-272.

12. Hoffmann J, Vitale I, Buchmann B, et al. Improved cellular pharmacokinetics and pharmacodynamics underlie the wide anticancer activity of sagopilone. Cancer Res. 2008;68(13):5301-5308.

13. Lee FY, Borzilleri R, Fairchild CR, et al. BMS-247550: a novel epothilone analog with a mode of action similar to paclitaxel but possessing superior antitumor efficacy. Clin Cancer Res. 2001;7(5):1429-1437.

14. Liberati A, Altman DG, Tetzlaff J, et al. The PRISMA statement for reporting systematic reviews and meta-analyses of studies that evaluate health care interventions: explanation and elaboration. J Clin Epidemiol. 2009;62(10):e1-e34.

15. Colombo N, Kutarska E, Dimopoulos M, et al. Randomized, openlabel, phase III study comparing patupilone (EPO906) with pegylated liposomal doxorubicin in platinum-refractory or -resistant patients with recurrent epithelial ovarian, primary fallopian tube, or primary peritoneal cancer. J Clin Oncol. 2012;30(31):3841-3847.

16. McMeekin S, Patel R, Verschraegen C, et al. Phase I/II study of sagopilone (ZK-EPO) plus carboplatin in women with recurrent platinumsensitive ovarian cancer. Br J Cancer. 2012;106(1):70-76.

17. Tsimberidou AM, Lewis N, Reid T, et al. Pharmacokinetics and antitumor activity of patupilone combined with midazolam or omeprazole in patients with advanced cancer. Cancer Chemother Pharmacol. 2011;68(6):1507-1516.

18. Rustin G, Reed N, Jayson GC, et al. A phase II trial evaluating two schedules of sagopilone (ZK-EPO), a novel epothilone, in patients with platinumresistant ovarian cancer. Ann Oncol. 2011;22(11):2411-2416.

19. Chuang E, Wiener N, Christos P, et al. Phase I trial of ixabepilone plus pegylated liposomal doxorubicin in patients with adenocarcinoma of breast or ovary. Ann Oncol. 2010;21(10):2075-2080.

20. De Geest K, Blessing JA, Morris RT, et al. Phase II clinical trial of ixabepilone in patients with recurrent or persistent platinum- and taxane-resistant ovarian or primary peritoneal cancer: a Gynecologic Oncology Group study. J Clin Oncol. 2010;28(1):149-153.

21. Ten Bokkel Huinink WW, Sufliarsky J, Smit WM, et al. Safety and efficacy of patupilone in patients with advanced ovarian, primary fallopian, or primary peritoneal cancer: a phase I, open-label, dose-escalation study. J Clin Oncol. 2009;27(19):3097-3103.

22. Forster M, Kaye S, Oza A, et al. A phase IB and pharmacokinetic trial of patupilone combined with carboplatin in patients with advanced cancer. Clin Cancer Res. 2007;13(14):4178-4184.

23. Hensley ML, Dizon D, Derosa F, et al. A phase I trial of BMS-247550 (NSC\# 710428) and gemcitabine in patients with advanced solid tumors. Invest New Drugs. 2007;25(4):335-341.

24. Rubin EH, Rothermel J, Tesfaye F, et al. Phase I dose-finding study of weekly single-agent patupilone in patients with advanced solid tumors. J Clin Oncol. 2005;23(36):9120-9129.

25. Zhuang SH, Agrawal M, Edgerly M, et al. A phase I clinical trial of ixabepilone (BMS-247550), an epothilone B analog, administered intravenously on a daily schedule for 3 days. Cancer. 2005;103(9):1932-1938.

OncoTargets and Therapy

\section{Publish your work in this journal}

OncoTargets and Therapy is an international, peer-reviewed, open access journal focusing on the pathological basis of all cancers, potential targets for therapy and treatment protocols employed to improve the management of cancer patients. The journal also focuses on the impact of management programs and new therapeutic agents and protocols on

Submit your manuscript here: http://www.dovepress.com/oncotargets-and-therapy-journal
26. Mani S, McDaid H, Hamilton A, et al. Phase I clinical and pharmacokinetic study of BMS-247550, a novel derivative of epothilone B, in solid tumors. Clin Cancer Res. 2004;10(4):1289-1298.

27. Abraham J, Agrawal M, Bakke S, et al. Phase I trial and pharmacokinetic study of BMS-247550, an epothilone B analog, administered intravenously on a daily schedule for five days. J Clin Oncol. 2003; 21(9):1866-1873.

28. Schmid P, Kiewe P, Possinger K, et al. Phase I study of the novel, fully synthetic epothilone sagopilone (ZK-EPO) in patients with solid tumors. Ann Oncol. 2010;21(3):633-639.

29. Arnold D, Voigt W, Kiewe P, et al. Weekly administration of sagopilone (ZK-EPO), a fully synthetic epothilone, in patients with refractory solid tumours: results of a phase I trial. Br J Cancer. 2009;101(8):1241-1247.

30. Aghajanian C, Burris HA 3rd, Jones S, et al. Phase I study of the novel epothilone analog ixabepilone (BMS-247550) in patients with advanced solid tumors and lymphomas. J Clin Oncol. 2007;25(9):1082-1088.

31. Awada A, Piccart MJ, Jones SF, et al. Phase I dose escalation study of weekly ixabepilone, an epothilone analog, in patients with advanced solid tumors who have failed standard therapy. Cancer Chemother Pharmacol. 2009;63(3):417-425.

32. Chen T, Molina A, Moore S, et al. Epothilone B analog (BMS-247550) at the recommended phase II dose in patients with gynecologic and breast cancers. J Clin Oncol. 2004;22(155 Suppl):2115.

33. Spriggs DR, Dupont J, Pezzulli S, et al. KOS-862 (epothilone D): phase 1 dose escalating and pharmacokinetic (PK) study in patients with advanced malignancies. Proc Am Soc Clin Oncol. 2003;22:894.

34. Smit WM, Sufliarsky J, Werner TL, et al. A phase II study evaluating the safety and efficacy of patupilone in patients with platinum refractory/ resistant ovarian, primary fallopian, or peritoneal cancer. J Clin Oncol. 2009;27(153):5563.

35. Calvert PM, O'Neill V, Twelves C, et al. A phase I clinical and pharmacokinetic study of EPO906 (epothilone B), given every three weeks, in patients with advanced solid tumors. Proc Am Soc Clin Oncol. 2001;20:429.

36. Hao D, Hammond LA, deBono JS, et al. Continuous weekly administration of the epothilone-B derivative, BMS247,550 (NSC710428): a phase I and pharmacokinetic (PK) study. Proc Am Soc Clin Oncol. 2002;21:411.

37. Piro LD, Rosen LS, Parson M, et al. KOS-862 (epothilone D): a comparison of two schedules in patients with advanced malignancies. Proc Am Soc Clin Oncol. 2003;22:539.

38. Dickson N, Peck R, Wu C, Burris H. Ixabepilone given weekly in patients with advanced malignancies: final efficacy and safety results of a phase I trial. J Clin Oncol. 2006;24(18 Suppl):2040.

39. Villalona-Calero M, Goel S, Schaaf L, et al. First-in-human phase I trial of a novel epothilone, KOS-1584. J Clin Oncol. 2006;24(18 Suppl):2003.

40. Mekhail T, Chung C, Holden S, et al. Phase I trial of novel epothilone B ana$\log$ BMS-310705 IV q 21 days. Proc Am Soc Clin Oncol. 2003;22:515.

41. Sessa C, Perotti A, Malossi A, et al. Phase I and pharmacokinetic (PK) study of the novel epothilone BMS-310705 in patients (pts) with advanced solid cancer. Proc Am Soc Clin Oncol. 2003;22:519.

42. Wozniak KM, Nomoto K, Lapidus RG, et al. Comparison of neuropathyinducing effects of eribulin mesylate, paclitaxel, and ixabepilone in mice. Cancer Res. 2011;71(11):3952-3962.

43. O'Reilly T, McSheehy PM, Wenger F, et al. Patupilone (epothilone B, EPO906) inhibits growth and metastasis of experimental prostate tumors in vivo. Prostate. 2005;65(3):231-240.

patient perspectives such as quality of life, adherence and satisfaction. The manuscript management system is completely online and includes a very quick and fair peer-review system, which is all easy to use. Visit http://www.dovepress.com/testimonials.php to read real quotes from published authors. 